\title{
Diagnosis of autoimmune neutropenia in a 10-month-old boy - a case report
}

\author{
KATARZYNA ZDANOWICZI, URSZULA DANILUK ${ }^{1}$, ELENA JEWSIEJENKO', \\ MILENA KRASNODĘBSKA ${ }^{l}$, RADOSŁAW MOTKOWSKI ${ }^{2}$, DARIUSZ MAREK LEBENSZTEJN ${ }^{l}$
}

'Department of Pediatrics, Gastroenterology, Hepatology, Nutrition and Allergology, Medical University of Bialystok, Poland ${ }^{2}$ Department of Pediatrics, Rheumatology, Immunology and Metabolic Bone Diseases, Medical University of Bialystok, Poland

\begin{abstract}
Neutropenia, congenital or acquired, is related to impaired granulocyte production in the bone marrow or increased destruction by antibodies. Autoimmune neutropenia of infancy (AIN) is associated with the occurrence of antineutrophil antibodies. AIN is the most common cause of neutropenia in infants and young children. However, its incidence is low. Detection of anti-neutrophil antibodies is an important step in confirming the diagnosis of AIN, although their detection is difficult due to low titer and poor avidity. In differential diagnosis, another cause of neutropenia should be considered, such as a drug-induced mechanism, viral infection, autoimmune and metabolic disease, hematological conditions or immune deficiency syndromes. Despite the benign course of AIN, serious infectious complications can occur. Spontaneous remission of neutropenia was observed in 95\% of patients during 24 months of follow-up. We present a case of a 10-month-old boy with deafness, heart defect and Morgagni-Larrey hernia diagnosed in our department because of formation of a skin abscess due to autoimmune neutropenia.
\end{abstract}

Key words: children, neutropenia, autoimmune disease, immunology, anti-human neutrophil antigen-1.

(Cent Eur J Immunol 2021; 46 (1): 118-120)

\section{Introduction}

Neutrophils play an important role as part of the innate immune system, and their reduced number may be associated with an impaired immune response. The term neutropenia is used to define a reduction in absolute neutrophil count (ANC) below 1500/ $\mu$, which may increase the risk of life-threatening infections, especially in the case of agranulocytosis $(\mathrm{ANC}<100 / \mu \mathrm{l})$ [1]. Neutropenia, congenital or acquired, is associated with impaired production of granulocytes in the bone marrow or increased peripheral destruction. Autoimmune neutropenia is caused by destruction due to autoantibodies directed against various neutrophil-specific antigens (HNA) [2].

We present a 10-month-old boy admitted to our department because of severe irritability with skin abscess formation and neutropenia.

\section{Case report}

A 10-month-old male infant with strong anxiety observed for one day was admitted to our department. Past medical history revealed surgery at the age of 8 months due to a Morgagni-Larrey hernia. In addition, the child was under the care of a cardiology outpatient clinic (ventricular septal defect type 2) and ENT outpatient clinic (bilateral sensorineural hearing loss). There were no abnormalities in complete blood counts and routine biochemical laboratory tests performed two and four months prior to hospitalization. The family history was insignificant. Physical examination revealed pale skin, postoperative scars, Mongolian spot in the sacral region, flat forehead, broad nasal bridge with a tendency to form epicanthal folds, single transverse palmar creases, persistent fetal pads, aphthous stomatitis, umbilical hernia and systolic murmur along the left border of the sternum. Preliminary results of laboratory tests showed agranulocytosis, monocytosis, anemia, and increase in C-reactive protein concentration. The results of laboratory tests are presented in Table 1 . There were no abnormalities of abdominal organs based on abdominal radiographs and ultrasound examination, except for umbilical hernia.

On the second day of hospitalization, the tender and painful mass about $2 \times 2.5 \mathrm{~cm}$, without splashing in the right submandibular area was palpated. Ultrasound examination of this area revealed the features of a forming abscess. An laryngological consultation was performed and initially empiric antibiotic therapy with amoxicillin and clavulanic acid was used, but without improvement. On the seventh day of hospitalization under general anesthe-

Correspondence: Katarzyna Zdanowicz, Department of Pediatrics, Gastroenterology, Hepatology, Nutrition and Allergology,

Medical University of Bialystok, Poland, e-mail: kazdanowicz@ gmail.com

Submitted: 3.01.2020; Accepted: 22.05.2020 
Table 1. Results of laboratory tests

\begin{tabular}{|c|c|c|c|c|}
\hline \multirow[t]{2}{*}{ Parameter } & \multicolumn{3}{|c|}{ Laboratory results } & \multirow[t]{2}{*}{ Normal range } \\
\hline & 4 months before admission & day of admission & discharge & \\
\hline White blood cells $[\mathrm{K} / \mu \mathrm{l}]$ & 10.67 & 6.57 & 5.19 & $5.0-12$ \\
\hline Neutrophils $[\mathrm{K} / \mu \mathrm{l}]$ & 2.7 & 0.131 & 0.155 & $1.5-7$ \\
\hline Monocytes $[\mathrm{K} / \mu \mathrm{l}]$ & 0.84 & 1.905 & 1.089 & $0.3-0.82$ \\
\hline Hematocrit [\%] & 32.8 & 24.5 & 35.5 & $34-40$ \\
\hline Mean corpuscular volume [fl] & 72.5 & 75.1 & 75.9 & $75.00-86.00$ \\
\hline Hemoglobin $[\mathrm{g} / \mathrm{dl}]$ & 10.8 & 8.0 & 12.2 & $11.0-14.0$ \\
\hline Platelet $[\mathrm{K} / \mu \mathrm{l}]$ & 394 & 685 & 350 & $140-450$ \\
\hline C-reactive protein $[\mathrm{mg} / \mathrm{l}]$ & & 13.56 & 4.27 & $0-5$ \\
\hline Creatinine $[\mathrm{mg} / \mathrm{dl}]$ & & \multicolumn{2}{|c|}{0.26} & $0.17-0.42$ \\
\hline Alanine transferase [U/l] & & \multicolumn{2}{|c|}{12} & $0-29$ \\
\hline Procalcitonin [ng/ml] & & \multicolumn{2}{|c|}{0.06} & $<0.5$ \\
\hline Triglycerides [mg/dl] & & \multicolumn{2}{|c|}{110} & $35-150$ \\
\hline Total cholesterol [mg/dl] & & \multicolumn{2}{|c|}{120} & $120-200$ \\
\hline Immunoglobulin G [g/l] & & \multicolumn{2}{|c|}{11.00} & $3.50-11.80$ \\
\hline Immunoglobulin $\mathrm{M}$ [g/l] & & \multicolumn{2}{|c|}{0.84} & $0.36-1.04$ \\
\hline Immunoglobulin A [g/l] & & \multicolumn{2}{|c|}{0.78} & $0.36-1.65$ \\
\hline Glucose [mg/dl] & & \multicolumn{2}{|c|}{76} & $60-100$ \\
\hline Lipase [U/1] & & \multicolumn{2}{|c|}{16} & $13-60$ \\
\hline Ammonia $[\mu \mathrm{mol} / 1]$ & & \multicolumn{2}{|c|}{36.3} & $21-50$ \\
\hline Lactate $[\mathrm{mmol} / \mathrm{l}]$ & & \multicolumn{2}{|c|}{1.59} & $0.50-2.20$ \\
\hline Uric acid $[\mathrm{mg} / \mathrm{dl}]$ & & \multicolumn{2}{|c|}{4.6} & $1.1-5.5$ \\
\hline Complement C3 [mg/dl] & & \multicolumn{2}{|c|}{166.00} & $90-180$ \\
\hline Complement C4 [mg/dl] & & \multicolumn{2}{|c|}{19.00} & $10-40$ \\
\hline Anti-CMV IgM & & \multicolumn{2}{|c|}{ negative } & negative \\
\hline Anti-EBV IgM, IgG & & \multicolumn{2}{|c|}{ negative } & negative \\
\hline HNA-1 antibodies & & \multicolumn{2}{|c|}{ positive } & negative \\
\hline Fecal elastase-1 $[\mu \mathrm{g} / \mathrm{g}]$ & & \multicolumn{2}{|c|}{473.3} & $>200$ \\
\hline
\end{tabular}

sia, a surgical incision and drainage of the abscess were performed. The pus culture indicated methicillin-sensitive Staphylococcus aureus (MSSA) infection. Due to agranulocytosis, immunological, genetic and hematological consultations were carried out to plan diagnostic tests to determine the cause of neutropenia. Epstein-Barr virus and cytomegalovirus infections were excluded. The lipid profile (total cholesterol and triglycerides), lactate and uric acid were within normal limits. The concentrations of elastase I and fat in the stool were normal; therefore exocrine pancreatic insufficiency was excluded. Urine organic acid analysis by gas chromatography-mass spectrometry (GC-MS) did not indicate inborn errors of metabolism. Immunophenotyping by flow cytometry was also performed and reference ranges for peripheral blood lymphocyte subsets were revealed. The immunoglobulin classes A, G, M, complement component 3 and complement 4 were within the normal range. Finally, anti-human neutrophil antigen-1 (HNA-1) anti- bodies were detected in serum using the granulocyte immunofluorescence test (GIFT), confirming an autoimmune neutropenia. Bone marrow biopsy was not performed due to parental disagreement. During hospitalization, the parents also refused to undergo genetic tests. After obtaining the culture result, antibiotic therapy was applied according to the antibiotic susceptibility testing. On the $14^{\text {th }}$ day of hospitalization, the boy was discharged in good general condition with the recommendation of antibiotic prophylaxis (amoxicillin $20 \mathrm{mg} / \mathrm{kg} / 24 \mathrm{~h}$ ) until the follow-up visit at the immunological clinic. Our patient did not develop infectious diseases after starting prophylaxis with amoxicillin during 6 months of follow-up despite ANC $<500 / \mu$ l.

\section{Discussion}

The causes of neutropenia are congenital disorders such as Shwachman-Diamond syndrome (SDS), Kostmann 
syndrome (KS), cyclic neutropenia $(\mathrm{CyN})$, glycogen storage disease type $1 \mathrm{~b}$ (GSD1b), primary immunodeficiency (PID) and organic acidemias. Acquired neutropenia may be induced by drugs, infections, leukemia, and autoimmune diseases. An example is autoimmune neutropenia of infancy (AIN), which was diagnosed in our patient [2].

The prevalence of AIN in children is $1: 100,000$, making it one of the most common causes of neutropenia in the pediatric population [2]. However, current research may suggest that previous data were underestimated based on the report of Farruggia et al. determining the incidence of AIN as $1: 6,300$ in children in Italy. AIN is diagnosed mainly at the age of 5-15 months and is more often observed in premature babies, which may be due to the immaturity of the immune system [3]. In most cases, the clinical course of AIN is mild. The most common manifestations of AIN in infants are upper respiratory tract infections, otitis media, pyoderma, fever of unknown origin, and gastroenteritis. More serious complications caused by Gram-positive and Gram-negative bacteria and fungal infections, such as pneumonia, sepsis or meningitis, are less common [4]. Our patient had a skin abscess due to a Gram-positive bacterial infection. The hematological abnormalities observed in AIN include isolated neutropenia or neutropenia in combination with anemia and/or thrombocytopenia and/or peripheral monocytosis; however, bone marrow examinations may show normal or increased cellularity with normal or low numbers of mature neutrophils. Detection of anti-neutrophil antibodies is a major step in confirming the diagnosis of AIN, although their detection is difficult due to low titer and avidity [5]. The presence of anti-neutrophil antibodies was first described by Lazerali in 1971 [6]. Currently, five human neutrophil antigens (HNA-1 to HNA-5) are included in the diagnostic procedure. In our case, anti-HNA-1 antibodies (Fc $\gamma$ RIIIb receptor expressed only on neutrophils) were detected. HNA-1a and HNA-1b are the most common isoforms of this antigen [7]. The origin of the antibodies against HNA is unknown. Their synthesis may be associated with the phenomenon of molecular mimicry of infectious agents, especially parvovirus B19 (B19) [4]. The change of endogenous antigens may also be a consequence of taking medications, abnormal HLA antigen expression or dysfunctions of the immunosuppressive response [2]. The patient's medical history should include questions about previous drug administration, symptoms of viral infections, e.g. Epstein-Barr virus (EBV), cytomegalovirus (CMV), $\mathrm{B} 19$, and history of hematologic/autoimmune diseases [8]. Interestingly, the count of neutrophils does not depend on the level of antibodies [5].

Spontaneous remission of neutropenia was observed in $95 \%$ of patients during 24 months of follow-up [4]. Treatment of AIN is individualized, depending on neutrophil levels, the age of the child, and the occurring infections. Due to the mild and self-limiting nature of the disease, rou- tine antibiotic prophylaxis is not recommended. However, in some studies, antibiotic prophylaxis was beneficial for patients with recurrent infections, especially skin infections $[9,10]$. The choice of therapy depends on the prevalence of specific bacteria and their resistance patterns in the environment [10]. Treatment of myeloid growth factors should be started after a previous bone marrow aspiration biopsy in children with severe infections or requiring surgical intervention [2]. High doses of intravenous immunoglobulin and corticosteroids may be effective in treating AIN in patients with life-threating infections. However, due to possible side effects, it is not standard treatment [5].

\section{Conclusions}

Autoimmune neutropenia of infancy, one of the most common causes of neutropenia, presents a diagnostic challenge in infants. Despite the benign course, serious infectious complications can occur. Due to numerous congenital anomalies and a lack of consent for an extended genetic test, the diagnosis of primary AIN in our patient will be finally confirmed after obtaining spontaneous remission of neutropenia.

\section{The authors declare no conflict of interest.}

\section{References}

1. Newburger PE, Dale DC (2013): Evaluation and management of patients with isolated neutropenia. Semin Hematol 50: 198206.

2. Capsoni F, Sarzi-Puttini P, Zanella A (2005): Primary and secondary autoimmune neutropenia. Arthritis Res Ther 7: 208-214.

3. Farruggia P, Fioredda F, Puccio G, et al. (2015): Autoimmune neutropenia of infancy: Data from the Italian neutropenia registry. Am J Hematol 90: E221-E222.

4. Bux J, Behrens G, Jaeger G, Welte K (1998): Diagnosis and clinical course of autoimmune neutropenia in infancy: analysis of 240 cases. Blood 91: 181-186.

5. Youinou P, Jamin C, Le Pottier L, et al. (2014): Diagnostic criteria for autoimmune neutropenia. Autoimmun Rev 2014; 3: 574-576.

6. Lalezari P, Jiang AF, Yegen L, Santorineou M (1975): Chronic autoimmune neutropenia due to anti-NA2 antibody. N Engl J Med 293: 744-747.

7. Veldhuisen B, Porcelijn L, Ellen van der Schoot C, de Haas M (2014): Molecular typing of human platelet and neutrophil antigens (HPA and HNA). Transfus Apher Sci 50: 189-199.

8. Autrel-Moignet A, Lamy T (2014): Autoimmune neutropenia. La Presse Médicale 43: e105-e118.

9. Kobayashi M, Sato T, Kawaguchi H, et al. (2003): Efficacy of prophylactic use of trimethoprim-sulfamethoxazole in autoimmune neutropenia in infancy. J Pediatr Hematol Oncol 25: 553-557.

10. Bruin M, Dassen A, Pajkrt D, et al. (2005): Primary autoimmune neutropenia in children: a study of neutrophil antibodies and clinical course. Vox Sang 88: 52-59. 